\title{
Comentarios críticos y cinco propuestas para pensar la migración en el momento actual
}

\author{
FEDERICO BESSERER
}

Critical Comments and

Five Proposals to Think Migration at the Current Time

Federico Besserer Departamento de Antropología, Universidad Autónoma Metropolitana-Iztapalapa, Distrito Federal, México fbesserer@hotmail.com

Desacatos 46, septiembre-diciembre 2014, pp. 88-105

\section{Cuatro viñetas \\ Cuatro vinetas}

n las siguientes páginas haré una revisión de los análisis que nos presentan los autores de los excelentes artículos que integran la sección "Saberes y razones” de este número de Desacatos. La estrategia que he seguido ha sido presentar primero cuatro viñetas, que surgen del trabajo de campo realizado por investigadores del Seminario de Estudios Transnacionales del Departamento de Antropología de la Universidad Autónoma Metropolitana-Iztapalapa. Estas viñetas me servirán de apoyo para construir un marco conceptual que propondré en la segunda sección del texto. Entonces, las propuestas de los autores serán revisadas conforme avanza el argumento para organizar un planteamiento que nos permita por un lado comprender los principales problemas que enfrenta en este momento la migración entre México y Estados Unidos, y por el otro articular los nuevos enfoques teóricos con los que contribuyen los autores para el análisis y la comprensión de la migración actualmente. Las posturas que encontramos en estos materiales gravitan entre una mirada pesimista de la realidad con énfasis en los retos que ofrecen las nuevas políticas migratorias y la violencia estructural y cotidiana en que transcurre la vida transmigrante. Por el otro lado están las miradas que ven indicios de cambio en las acciones de las comunidades y las asociaciones que conforman el ensamblaje social transnacional que se extiende entre los dos países.

I
Espero sentado en los sillones de una pequeña peluquería en los alrede-
dores de la ciudad de Barcelona y observo a un grupo de mujeres ves-
tidas a la usanza marroquí que hacen fila para llenar cubetas con agua.
Las llevarán a los departamentos del edificio de enfrente, que carecen
de servicios como agua y energía eléctrica, porque fueron desalojados 
durante el proceso instrumentado por los bancos de recuperación de hipotecas que los antiguos habitantes no pudieron pagar. Estas mujeres son parte de las familias "ocupas" que se instalaron en los departamentos deshabitados, hoy desempleadas, pues trabajaban en la industria de la construcción que se encuentra detenida en el marco de la crisis económica iniciada en 2008 y que todavía azota la economía de esta población migrante. El señor Mustafá termina de cortar el pelo a uno de sus vecinos que sale sin pagar de la peluquería y lleva su mano al corazón en señal de agradecimiento. Esta economía de la solidaridad se sostiene en parte por las "remesas" que llegan a Barcelona desde Marruecos. El sostenimiento del señor Mustafá en las conurbaciones de Barcelona, cuya esposa e hijos en edad escolar viven en las márgenes de la mediterránea ciudad de Tánger, proviene de la venta de una propiedad que tenían en aquella ciudad de entrada al continente africano. Esperanzados en que la economía se recupere, los habitantes de estas dos orillas urbanas separadas por una frontera acuática, pero articuladas en "la ciudad transnacional desde abajo", pagan los costos de recuperación del entramado urbano que forma el conjunto de "ciudades globales" europeas, entre las que Barcelona juega un papel destacado: antes como ciudad industrial, hoy llamada "ciudad del conocimiento” (Besserer, notas de campo, 2013).

\section{II}

Sandra vivía en una casa de autoconstrucción en la periferia urbana de la ciudad de México, literalmente en la última calle de la colonia Arboledas de Cuautepec. El teléfono que estaba a disposición de los vecinos en el estanquillo familiar que ella atendía era el punto de enlace de familias cuyas vidas se desarrollaban entre Arboledas y los edificios Forest Hills en la ciudad de Myrtle Beach, Carolina del Sur. El padre de Sandra organizaba para las empresas constructoras de la creciente ciudad turística estadounidense el trabajo de los vecinos de Arboledas, que seguían siendo sus vecinos ahora en Forest Hills. Sandra cursó exitosamente el high school en Myrtle Beach, pero tuvo que regresar a México porque no contaba con la residencia legal, indispensable para continuar con sus estudios universitarios. Mientras concluía su licenciatura en antropología en la ciudad de México, trabajaba en un call center donde sus saberes lingüísticos contribuyeron — por un salario menor al que recibiría en Myrtle Beacha la industria global del conocimiento. Este espacio de trabajo era una zona fronteriza - un hiperespacio- en la que implosionaban husos horarios diferentes - el del trabajador en México y el del cliente en Estados Unidos - y en el que se articulaban los saberes transnacionales de los márgenes urbanos de Arboledas con la clientela radicada en los Estados Unidos, a través de una cadena global de servicios. Así, mientras las políticas migratorias sitúan a un creciente número de retornados, deportados y deportables, a disposición de la cadena de producción global, las fronteras mantienen la desigualdad salarial que redunda en mayor ganancia para una de las industrias de mayor crecimiento en América Latina. Myrtle Beach fue uno de los condados donde la crisis económica de 2008 causó el mayor porcentaje de desempleo en Estados Unidos, que afectó en particular a la industria de la construcción y con ello a la familia de Sandra, lo que provocó que algunos de sus familiares regresaran a México. El lado mexicano de la calle Arboledas-Forest Hills absorbió los costos de la crisis global a través de la solidaridad familiar (Tafolla, 2014).

\section{III}

El Manhattan es un barrio de la población de Santa Cruz Tacache de Mina, situada en la región mixteca de Oaxaca. Los tenedores de los terrenos de 


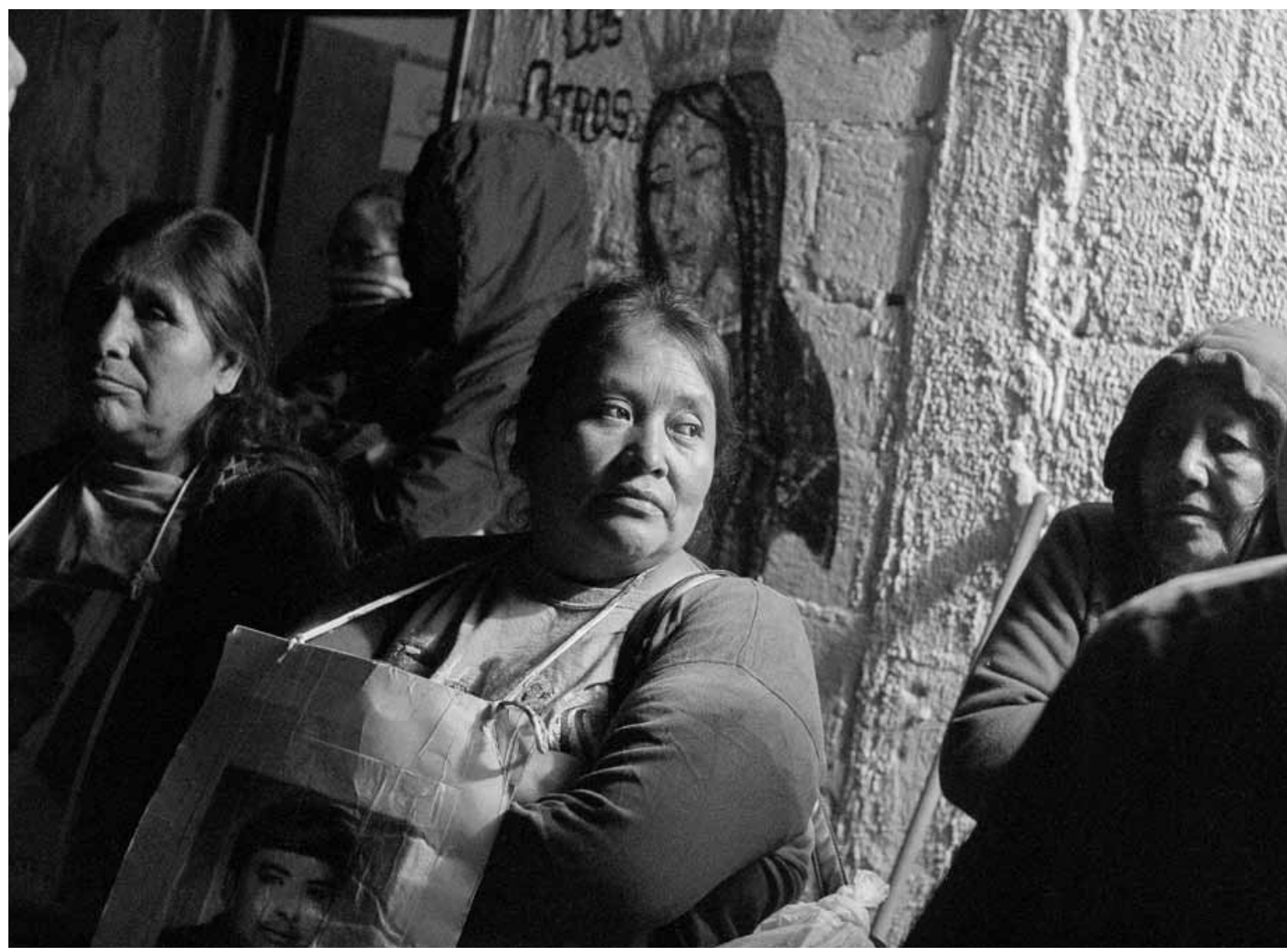

Prometeo Lucero - Caravana de madres centroamericanas, en búsqueda de familiares, Apizaco, Tlaxcala, 24 de octubre de 2014.

El Manhattan trabajan en las orillas de la ciudad de Nueva York, donde desarman automóviles que han salido de circulación. Su trabajo es indispensable para mantener en funcionamiento los vehículos de los trabajadores precarizados de la Gran Manzana que laboran en los servicios de cuidado de niños y ancianos, de limpieza y en restaurantes del otro Manhattan, el de la ciudad global. Las proyecciones del Instituto Nacional de Estadística y Geografía (INEGI) auguraban un decrecimiento constante en la población de Tacache de Mina, pero no sucedió así. En un artículo de próxima aparición, Lilia Solís reporta que la población en el pueblo de origen de su familia ha aumentado significativamente durante los últimos años debido a las deportaciones que se han hecho desde Nueva York. Muchos no se van, otros regresaron a México y decidieron quedarse aquí. En Tacache ha crecido el número de taxistas que ofertan sus servicios. Esta ocupación, que parece adecuada para los retornados en edad adulta, no parece tener la capacidad de absorber las necesidades de ocupación de esta creciente población mayor de edad en la comunidad. Tacache, como muchas otras localidades de la Mixteca oaxaqueña, enfrenta un proceso demográfico preocupante de envejecimiento de la población. En las décadas anteriores trasladaron su bono demográfico a Estados Unidos y ahora tendrán que absorber los costos del cuidado de la población mayor que regresa (Solís, en prensa). 
En El Salvador, a la edad de 12 años, Ros fue obligada bajo amenaza de muerte a vivir con un joven marero que era un vecino del barrio. Los esfuerzos por liberarla terminaron en fracaso con la muerte de varios miembros de la familia, que vivía de las remesas que su madre enviaba desde Tijuana, de donde regresó cuando se enteró de la situación. Un año y medio después Ros logró escapar y se trasladó con su madre a México, donde fueron detenidas en la Estación Migratoria Siglo XXI, tras lo cual decidieron solicitar refugio. La madre intentó suicidarse y fue conducida fuera del país, pero Ros decidió continuar en el encierro con la esperanza de conseguir la estancia legal y buscar que su madre fuera aceptada en México también de manera legal. La madre regresó a la frontera entre Chiapas y Guatemala, donde fue atrapada por una red de trata de personas. Por ser menor de edad, el Estado mexicano puso a Ros al cuidado de una instancia no gubernamental. Meses después recibió la documentación para permanecer en México y pasado algún tiempo pudo reunirse con su madre, que también obtuvo la residencia legal. Sandra Guillot registra este caso en su tesis de licenciatura y explica que se reencontró con Ros y con su mamá en la ciudad de México, donde viven en condiciones de pobreza. Ros no ha continuado con sus estudios y trabaja para contribuir al ingreso de un hogar precario en la Delegación Iztapalapa.

\section{Cinco propuestas para pensar la migración en el momento actual}

Los casos que he descrito me permiten hacer referencia a algunos procesos económicos, demográficos y políticos que subyacen y afectan a la migración entre México y Estados Unidos. Me interesa mostrar que estos procesos generales no son privativos de la situación mexicana, forman parte de procesos de mayor escala. A partir de las viñetas anteriores trataré de mostrar etnográficamente cómo estos procesos generales se expresan en la cotidianidad de los migrantes.

\section{CAMBIO ECONÓMICO Y CRISIS GLOBAL}

Los casos de la migración marroquí a España, de la comunidad transnacional de Tacache de Mina y de la migración entre la ciudad de México y Carolina del Sur me permiten sostener que como telón de fondo de los cambios que percibimos en la migración está un fenómeno económico de escala mundial: un nuevo modo de acumulación que manifestó su primera crisis de gran magnitud en 2008. David Harvey (2008) propuso que se trataba de una crisis que se expresaba fundamentalmente en el ámbito urbano y que había traído aparejado el endurecimiento de lo que Nicholas de Genova denominó un "régimen de deportación” (De Genova y Peutz, 2010). Ésta es la primera gran crisis de un nuevo momento del capitalismo que cambió en las últimas décadas del siglo xx para dar paso a una configuración que, según el marco analítico que lo estudie, ha sido llamado "posfordismo", "capitalismo tardío" o etapa de "acumulación flexible". Entre las características más notorias de esta nueva configuración está la globalización de los procesos productivos — antes concentrados en grandes unidades fabriles o agrícolas - que da origen a cadenas globales de producción dispersas en la geografía global. Este cambio estuvo relacionado, entre otras cosas, con el desarrollo de la tecnología informática y de comunicación que hizo posible que la dispersión geográfica fuese compensada por el intercambio de grandes cantidades de información de manera instantánea. En el caso de la agricultura, este proceso de globalización se vio favorecido por la conformación de cadenas frigoríficas que garantizaron el transporte de 
productos entre hemisferios y zonas climáticas. En la industria se percibió como una era postindustrial en los países desarrollados y como el auge de la industria maquiladora en otras regiones del mundo. En el mundo de las finanzas surgió un aparato financiero que, disperso en ciudades alrededor del mundo, funciona las 24 horas del día y contribuye a la formación de lo que se conoce como la "ciudad global”. Este cambio fue paralelo a la pérdida de la centralidad de la industria pesada y al surgimiento de una economía enfocada en la producción ligera y la generación de servicios. Esta modificación fue notoria en especial en la transformación del paisaje urbano: en las ciudades que antes habían sido el epítome de la producción fabril, la actividad predominante es ahora la provisión de servicios y el manejo de información. El nuevo sistema productivo trajo también una nueva organización del trabajo, caracterizado por su flexibilidad, la precariedad en los sistemas de contratación y los nuevos mecanismos de control. Los casos descritos en las viñetas dan testimonio de la demanda de ejércitos de trabajadores de la construcción para la transformación urbana en las grandes ciudades del mundo, como Barcelona; del surgimiento de los nuevos empleos en las cadenas globales de la información y los servicios, como los call centers de la ciudad de México, y el crecimiento de la economía informal que da soporte a los trabajadores precarizados del nuevo sistema, como en el caso de los dos Manhattan. En todos, la disponibilidad de fuerza de trabajo se relaciona con grandes movimientos de población que después de casi medio siglo dio lugar a vidas transnacionales que, como el capital, construyó una continuidad entre diversas localidades del mundo. La crisis de 2008 fue resultado de prácticas financieras desmedidas asociadas al creciente mercado inmobiliario de las grandes ciudades del mundo. Esta crisis impactó directamente a los empleados del sector de la construcción — como la familia de Sandra-, sobre quienes habían adquirido inmuebles —como los habitantes marroquíes de las inmediaciones de Barcelona-y sobre los lugares de origen de estos urbanitas marginados — como los habitantes de la ciudad de México y la ciudad norafricana de Tánger.

Si bien los artículos en este número de Desacatos enfatizan en los acontecimientos de 2001, de los que se derivaron nuevas políticas de seguridad que afectaron a la migración, es importante tomar en cuenta el argumento de Guillermo Alonso Meneses, que sostiene que ya desde la década de 1990 inicia un problema de desempleo en Estados Unidos que implica una retórica antiinmigrante. Durante esa misma década se ponen en marcha en la frontera operativos como "Operation Blocade", "Hold the Line", "Gatekeeper" y "Safeguard", y se promueve una legislación sobre migración que propone la existencia de facetas criminales al interior del proceso migratorio. El aumento de los recursos para el reforzamiento fronterizo comienza en esa década y desde 1995 se duplica el número de guardias fronterizos. Así, las acciones y políticas contra la migración inician antes de 2001. Después de esta fecha se profundizó el discurso antiinmigrante y se construyó una legislación que permitió el incremento del presupuesto para el control fronterizo. Se construyó lo que Alonso Meneses llama "la frontera-gulag", pero el crecimiento sustantivo de las deportaciones ocurrió durante el bienio 2008-2009, es decir, en el contexto de la crisis económica y la intensificación del desempleo en Estados Unidos. Las políticas fronterizas se entreveraron con el discurso racista existente y se transformaron en un instrumento de "limpieza étnica", que puede asociarse con una selección de clase - una "cirugía de extirpación socioeconómica”- - Si bien resulta difícil establecer este discurso, sí ha tenido como consecuencia un incremento total de las deportaciones en los últimos años, opera sobre un número mayor de personas por su efecto "disuasivo" relacionado, entre otras cosas, con el aumento de la violencia en las acciones de la patrulla fronteriza. 
Yerko Castro Neira propone una explicación para la relación economía-etnicidad y la manera en que operan los discursos racistas en un marco económico en Estados Unidos. Dice Castro que existen "campos económicos", como algunos sectores de la agricultura, que son zonas de excepción en las que prevalece la precariedad y la ausencia de derechos laborales. El caso de los trabajadores mixtecos ilustra cómo los trabajadores quedan atrapados en una “injusticia y precariedad transnacional” por estar sujetos a procesos de exclusión tanto en sus lugares de origen como de destino. Desde el inicio del siglo xx hasta las nuevas fórmulas de producción en el marco de las cadenas globales, varios grupos étnicos han sido incorporados a las tareas productivas de estas zonas como "clase obrera deslocalizada" y sustituidos por otros grupos étnicos en momentos de crisis económica o de aumento en la organización por la búsqueda de derechos. Se les presenta como una “amenaza cultural” y se genera una dinámica que se ha definido como "reemplazo étnico". Las relaciones de producción se vinculan con una "economía emocional", propone el autor, en la que los "sentimientos culturales", como el rechazo, el odio racial y de clase, son parte de la economía política de los sentimientos, que son la fuerza fundamental que modela las dinámicas entre economía y migración. Hay una dialéctica en esta economía emocional que tiene como contraparte la indignación de los trabajadores ante la falta de derechos, el racismo y la explotación.

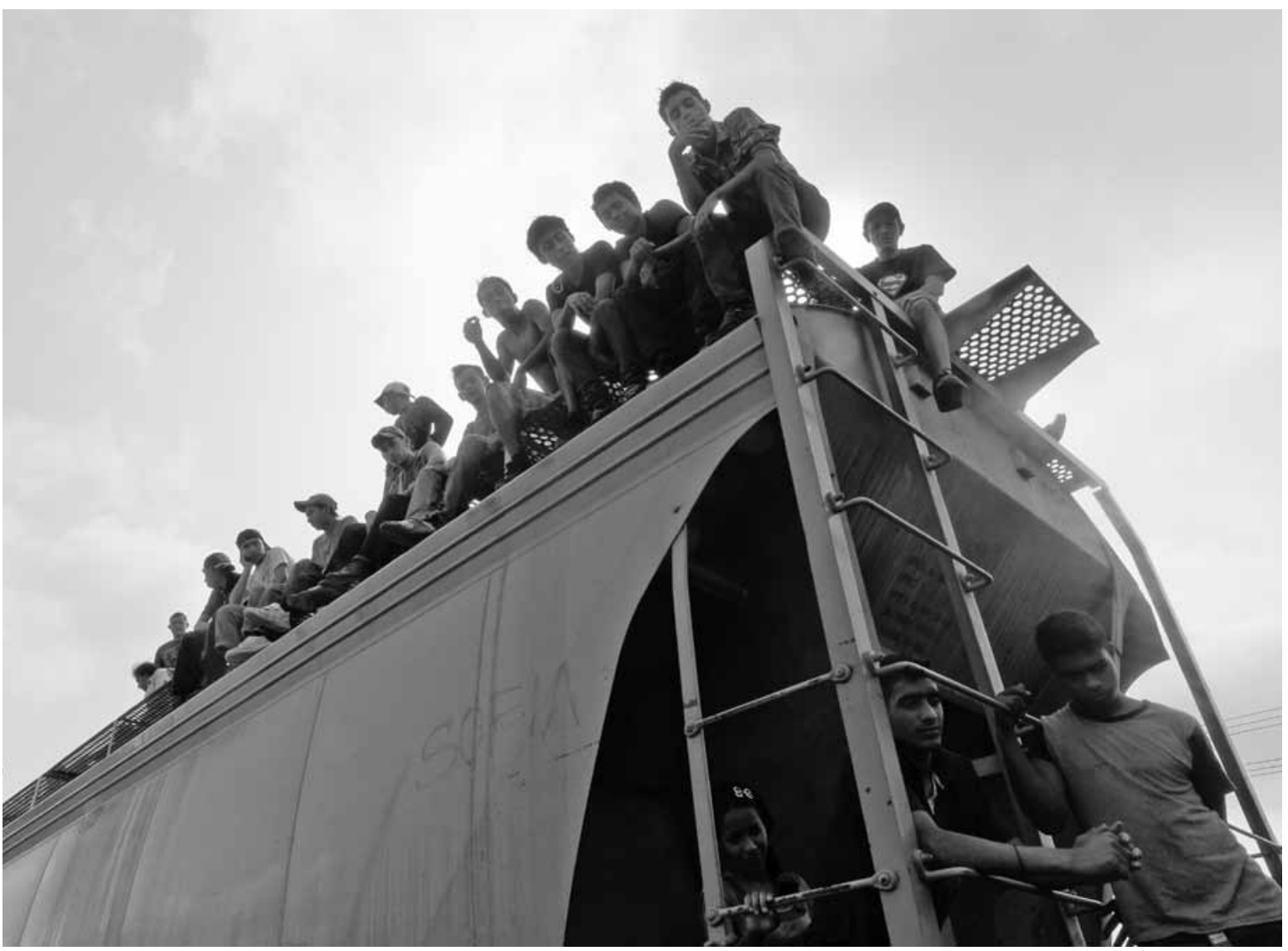

Prometeo Lucero • Migrantes abordan el ferrocarril de carga, apodado “La Bestia”, en la estación ferroviaria de Tenosique, Tabasco. 
La criminalización de los migrantes en los años recientes y las acciones de deportación deben ser entendidas en el contexto amplio de una fórmula de deportación étnica que tiene como trasfondo dinámicas económicas y estrategias que operan en momentos de crisis, como el proceso de repatriación de la década de 1930 y las deportaciones posteriores a 2008. La crisis de 2008 se expresó fundamentalmente como una crisis urbana. Uno de los elementos distintivos de las acciones relacionadas con las nuevas políticas de migración es que, según Guillermo Alonso Meneses, aumentaron las "redadas" en las ciudades. Esto sugiere la extensión de la economía emocional planteada por Castro al medio urbano, donde el miedo a la deportación —como un incitador al "regreso voluntario" o a la disminución de la emigración - se constituye como parte de un "régimen sentimental" que se suma a los dispositivos de movimientos de población con propósitos económicos.

\section{BIOPODER Y DINÁMICAS DEMOGRÁFICAS}

Esta crisis político-económica se relaciona también con una dimensión demográfica que debe ser analizada y que no parece tratarse a profundidad en este número de Desacatos. Desde finales del siglo xx se hizo notar que había una transferencia del así llamado "bono demográfico" de los países antes receptores de migración hacia las grandes metrópolis. En los países europeos y americanos receptores de migrantes estuvo relacionado con un proceso de "transición demográfica" en el que se estrechaba la base de la pirámide de población, mientras que en los países expulsores la población joven ensanchaba la base de la pirámide demográfica. Al fenómeno resultante de la transferencia de población por vía de la migración — de personas con situaciones migratorias precarias en muchos casos- se le llamó “convergencia demográfica" (Conapo, 2002). En el caso español, la transición demográfica tenía en su origen la reducción del crecimiento natural de la población. En el caso estadounidense, se sumaba el envejecimiento de un grupo etario denominado "baby boom". En ambos países la población migrante joven ayudó a construir el cambio urbano y a cubrir la demanda de fuerza de trabajo en el sector de servicios, incluyendo el cuidado de la población de mayor edad. Esto ha generado procesos transnacionales, como las “cadenas de cuidados", según le nombró Gioconda Herrera, que describió casos de personas que viajaron a España para trabajar como cuidadores y dejaron a sus propios hijos y padres bajo el cuidado de familiares en el Ecuador (Herrera, 2005). Esta situación se describe en la viñeta de Tacache de Mina para la migración México-Estados Unidos. Otras cadenas, como las de construcción (Wence, en prensa), avituallaron la demanda en sectores crecientes de la economía. Esta base poblacional migrante joven, a decir de Virgilio Partida (Conapo, 2002), contribuye también al sostenimiento del sistema de seguridad social de los países de llegada. En reciprocidad, el beneficio para las sociedades de origen de los migrantes debía ser que se multiplicaran las posibilidades de trabajo, lo que estrecharía la pirámide de edad, mientras la ampliación de la parte superior de la columna demográfica podría deberse, entre otros motivos, al posible retorno de personas de más edad. Con base en esta hipótesis realizamos trabajo de campo entre 2003 y 2005 en comunidades rurales en la región mixteca oaxaqueña de México. Encontramos dos fenómenos escalofriantes. El primero fue que los censos de las poblaciones de origen mostraban efectivamente la ausencia de jóvenes en edad laboral, lo que transformaba sus pirámides de edad en gráficas con forma de relojes de arena y explicaba que la mayoría de los hogares estuvieran compuestos por abuelos y nietos cuyo sustento provenía en gran medida de las remesas familiares. En aquellas localidades los proyectos de desarrollo habían fracasado por 
falta de personas jóvenes que realizaran las tareas de la producción (Gil, 2006). Estos datos coinciden con los resultados del Censo de Población y Vivienda 2010 (INEGI, 2011b), que revelan que Oaxaca es el estado con mayor índice de dependencia - mayor número de niños y personas de mayor edad por miembros que trabajan en la unidad-. Encontramos también que las mismas comunidades estaban levantando censos totales de población de las comunidades transnacionales, hacían un conteo total de la población que decía pertenecer a ellas para incorporar en el listado a todos los miembros de la comunidad en México o en Estados Unidos. El resultado fue que la comunidad en su conjunto estaba inmersa también en el proceso de transición demográfica. Los miembros de la comunidad reconocieron dos problemas derivados de esta información. El primero es que en un futuro mediato la población ahora infantil —en unos años económicamente activa - tendría dificultades para sostener un número creciente de personas de mayor edad, representado en la parte superior de la columna demográfica. El segundo problema es que esta población mayor regresaba a las poblaciones en Oaxaca. Esta voluntad de regreso explica, entre otras cosas, el gran número de casas desocupadas construidas por los jóvenes migrantes en las poblaciones de origen, como en el caso de El Manhattan en Tacache de Mina. Las comunidades empezaban a organizarse para atender el proceso de "retorno" que había iniciado, no así el estado. Este regreso a la comunidad de origen de las personas de mayor edad era una situación alarmante en los primeros años de este siglo y vino a acentuarse con las políticas públicas de 2001 y con el proceso de deportación posterior a 2008. La información demográfica indica que los deportados son de mayor edad que el promedio de la población migrante (Fundación BBVA, 2012). Según información del Consejo Nacional de Población, los retornados para todo el país en el último periodo — sean deportados o que volvieron por voluntad propia - son en promedio de mayor edad que los retornados de hace una década (Serrano, 2014). En otras palabras, el proceso de "convergencia demográfica" se está invirtiendo y ahora la migración de retorno está compuesta de personas que pronto estarán, o ya están, desocupadas. El aumento del número de taxistas en Tacache de Mina es muestra de este proceso (Solís, en prensa). La crisis económica y sus consecutivas deportaciones se suman entonces a un proceso demográfico de mayor alcance, que intensifica el problema para las comunidades que absorben los costos de una crisis de acumulación y de una tendencia demográfica en el país vecino, lo que genera una contingencia de corte transnacional, esta vez en territorio mexicano.

La contraparte de este proceso puede hallarse en la contribución de Patricia Fortuny y Marie Friedmann Marcquardt para este número de Desacatos. Las autoras presentan a las iglesias como espacios en los que convergen la generación de migrantes nacidos en México y las nuevas generaciones de mexicanos nacidos en Estados Unidos. Estos "nuevos mexicanos”, muchos de los cuales obtendrán la ciudadanía estadounidense, son parte de la base demográfica que sustentará a la sociedad, a la economía y al sistema de retiro del país vecino. Sujetos a las nuevas políticas de la diferencia y la exclusión en territorio estadounidense, su participación en los procesos sociales de las comunidades de origen de sus padres depende de procesos culturales e identitarios. Estos procesos conforman un nuevo campo de trabajo para la antropología y la demografía que ayudarán a comprender las dinámicas desde el punto de vista de las comunidades transnacionales y que requieren a su vez instrumentos propios para su estudio, como los "censos transnacionales" que las propias comunidades ya realizan, para la planeación del desarrollo comunitario, incluyendo la migración y los nuevos problemas y retos que significa (Gil, 2006). 
UN NUEVO ENSAMBLAJE SOCIAL TRANSNACIONAL Y LA ABSORCIÓN DE LOS COSTOS DE LA CRISIS

En el último medio siglo de migraciones entre México y Estados Unidos se ha generado un nuevo ensamblaje social (Actor-Network Theory, 2010) compuesto por lo que algunos analistas han llamado “espacios sociales transnacionales" (Pries, 2002). Son entramados de relaciones sociales que se expanden entre varios países a partir de las acciones de personas o colectivos migratorios que al mismo tiempo que establecen vínculos con el lugar de llegada, conservan sus relaciones con el país y la comunidad de salida. Pueden ser redes migratorias (Herrera, Calderón y Hernández, 2006), circuitos migratorios transnacionales (Rouse, 1991) o comunidades transnacionales (Kearney y Nagengast,
1989), y varían en la densidad y durabilidad de las relaciones que los constituyen, así como en la complejidad de las instituciones que los articulan, que pueden ir desde el parentesco hasta sistemas complejos de gobierno, como en el caso de las comunidades indígenas. Esta nueva sociología involucra también a quienes no han partido, ya sea porque no migraron o porque nacieron en el lugar que la generación que les precede consideraba un "destino". Las primeras investigaciones en nuestro país se enfocaron en el estudio de comunidades de origen rural (Kearney y Nagengast, 1989; Besserer, 1986; Gil, 2006: Besserer y Kearney, 2006; Castro, 2009; Oliver y Torres, 2012; Wence, 2012; Aguilar, 2012; Moctezuma, 2011; Rivera, 2012), y más recientemente en espacios sociales transnacionales urbanos (Hirai, 2009; Pizarro, 2010; Alba, 2014; Reyes, 2014;

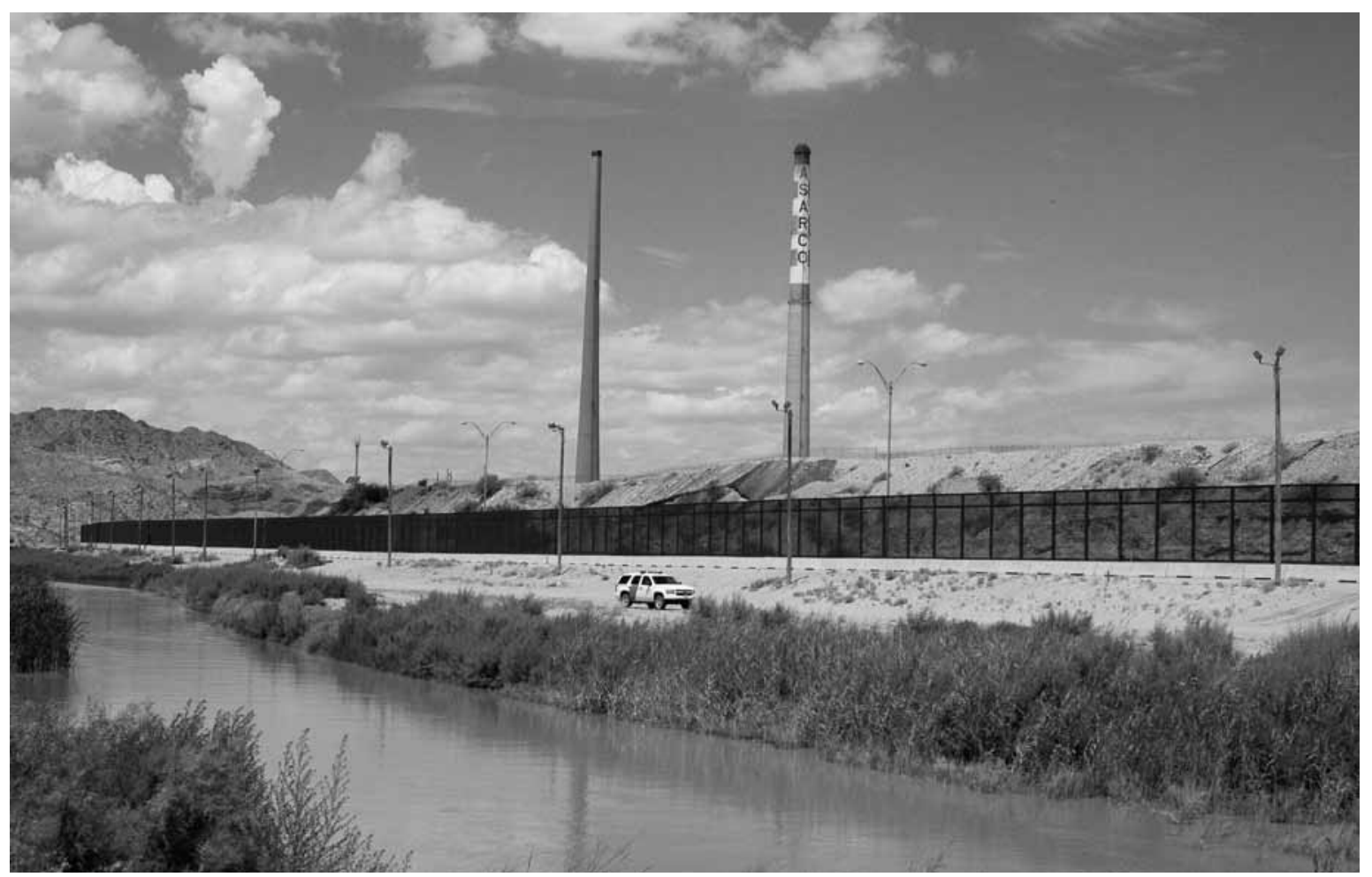

Prometeo Lucero Frontera Ciudad Juárez-El Paso. 
Tafolla; 2014; Lara, 2014). Las comunidades rurales de origen mexicano parecen sostenerse a partir de una base económica que comparten, como el territorio o una red laboral transnacional, y pueden tener un alto grado de cohesión y organización política, como sucede con los sistemas de cargos de las comunidades transnacionales de origen indígena. Pero las comunidades transnacionales varían. Las de origen aridoamericano tienden a estar abiertas a incorporar sujetos ajenos a la comunidad en sus estructuras económicas y tener una organización política poco institucionalizada, mientras que las comunidades indígenas suelen cerrarse a compartir los recursos materiales y pueden tener un sistema de organización político muy complejo y transnacionalizado. Por eso hemos hablado, según el modelo de Wolf y Mintz (1957), de comunidades laborales transnacionales "abiertas no corporadas" y "cerradas corporadas", como extremos en una gran diversidad de tipos de comunidad de origen rural (Besserer y Kearney, 2006; Solís, en prensa). Podemos identificar asimismo espacios sociales urbanos transnacionales con un alto grado de institucionalidad y densidad organizativa — como la comunidad de Tacache de Mina- o lo contrario - como la calle transnacional de la viñeta de Sandra Tafolla-. En todos los casos encontramos formas específicas de pertenencia social, pero en algunos hay sistemas de gobierno que mantienen una relación no sólo de pertenencia sino de "ciudadanía" con los miembros de la comunidad, como ellos mismos suelen llamarla. En algunos casos observamos gran centralidad en un gobierno comunitario anclado en los territorios de la comunidad histórica (Aguilar, 2012) y en otros detectamos sistemas de gobierno des-centrados con una compleja estructura de toma de decisión política que involucra a colectivos afianzados en distintas latitudes, donde incluso residen autoridades comunitarias (Gil, 2006). Estas comunidades tienen "autoridades" y sistemas complejos de pertenencia y ciudadanía transnacional, difieren de las asociaciones de afiliación voluntaria o clubes, que por lo regular cuentan con miembros y liderazgos, y no con autoridades y ciudadanos. Las comunidades y espacios sociales urbanos transnacionales suelen tener fuertes mecanismos de articulación interna, por lo que algunas veces han sido denominados como "rizomáticos" —en referencia a la metáfora usada por Deleuze-, en tanto que las organizaciones de membresía disponen de una gran capacidad de vinculación con su entorno, por lo que responderían mejor al modelo "reticular" — referido por Kearney- (Besserer y Gil, 2008). Las comunidades y los espacios urbanos transnacionales pueden ser y son con frecuencia espacios de mucho conflicto, que atienden las necesidades de los transmigrantes - muchos carentes de respaldo en otros lugares por su condición migratoria-, que al mismo tiempo están fuertemente acosadas por diversos sistemas de extracción de recursos al punto de que su continuidad está en peligro constantemente (Wence, 2012). Tal vez Robert Smith tenga razón cuando propone que si bien antes existían comunidades dispersas territorialmente, la posibilidad de establecer relaciones de "simultaneidad" — a través del teléfono, como en la viñeta de la calle transnacional de Arboledas - las hace significativamente diferentes en las últimas décadas respecto de las formas de organización anteriores (Smith, 2008). En los hechos, las relaciones que se establecen no pueden ser "inmediatas", pues están mediadas no sólo por los viajes de retorno de las personas, sino por la circulación de bienes y comunicación que no puede ser pensada sin las tecnologías o "medios" que la facilitan. Por estas tres razones - el papel que juegan los objetos en la configuración de las comunidades y los espacios urbanos transnacionales, por la incertidumbre que deriva de las presiones y conflictos a que están expuestas y por la complejidad de los ensamblajes que establecen con otras formas de organización, como asociaciones, federaciones, organizaciones no gubernamentales y Estados- es que pensamos que el 
concepto de "agenciamiento" o "ensamblaje" propuesto por la teoría del actor-red (Actor-Network Theory, ANT) puede ayudar a comprender mejor estas cambiantes configuraciones transnacionales que no pueden estudiarse como sistemas rígidos, estrictamente solidarios, de relaciones que son sólo sociales (Actor-Network Theory, 2010). Para quienes viven estos entramados, el hecho mismo de construir el lazo social con una llamada telefónica representa un ingreso económico para un tercero, que puede incluir a la compañía telefónica, al establecimiento donde se compra la tarjeta para llamadas o al local con un teléfono fijo desde donde se pide una llamada de larga distancia. Por eso la construcción misma de esta realidad colectiva es un proceso de jerarquización, subordinación y expoliación. Esta situación sólo puede comprenderse si pensamos a los objetos como parte de los ensamblajes sociales. Son estos ensamblajes transnacionales — que están dentro del entramado de las tecnologías globales, constituidas dentro de la globalización y no al margen de ella- los que soportan ahora los costos de la crisis económica y los que responden ante el poder simbólico y real de las medidas de deportación sistemática del biopoder transnacional.

El trabajo que ha realizado Yerko Castro Neira en una comunidad de origen mixteco —a la que se refiere en su contribución en este número de Desacatos- es un ejemplo de las organizaciones comunitarias transnacionales (Castro, 2009). La muy densa y compleja dinámica política interna es de carácter fundamentalmente rizomático y se dirime entre ciudadanos transnacionales que viven en más de 200 localidades en México y Estados Unidos. Esta comunidad cuenta también con asociaciones en los lugares de llegada, en las que la filiación es por membresía y cuyas acciones tienen un sentido reticular con agendas que incluyen la vinculación con otras organizaciones de la sociedad civil. Estas asociaciones son similares a lo que Luis Escala-Rabadán llama "clubes" en su artículo, que muchas veces se agregan en federaciones y confederaciones. La dinámica diferente entre organizaciones rizomáticas y reticulares es importante. Mientras las comunidades se asocian a partir de sus aparatos políticos y autoridades, como se propuso hacerlo la Red Internacional Indígena Oaxaqueña, la dinámica reticular construye un tipo de entramado distinto, como en el caso del Frente Indígena de Organizaciones Oaxaqueñas, que participa activamente en la construcción de lo que Escala-Rabadán llama la "sociedad civil migrante". Es verdad que los clubes pueden fortalecer a las comunidades, y a la inversa, pero no es útil reducir las unas a los otros. Las comunidades transnacionales suelen asumir responsabilidad sobre sus ciudadanos y demandar participación económica y administrativa de los mismos, y los clubes por su parte contribuyen a menudo con las comunidades, sin un compromiso u obligación formal hacia ellas. Por otro lado, la demografía de las "comunidades transnacionales" es una plataforma numérica mucho más amplia que la membresía de los clubes. Un tercer tipo de organización es la que plantean Patricia Fortuny y Marie Friedmann Marquardt cuando describen con fineza etnográfica las iglesias y las comunidades que configuran sus feligresías, que frecuentemente vinculan a sujetos de un gran número de comunidades transnacionales y locales. Las iglesias son en sí mismas instituciones transnacionales con agendas propias, que rebasan fronteras y conforman "campos transnacionales de la fe". Siguiendo a Levitt y Glick (2004), podemos pensar que una persona puede ser ciudadano de una comunidad, miembro de una organización y feligrés de una iglesia. De igual manera son diferentes los papeles y atribuciones de la jerarquía religiosa, los líderes de las asociaciones y las autoridades comunitarias. Mantener la diferencia entre estos tres tipos de organización, sus organizaciones y bases sociales puede ser de utilidad para la construcción de una teoría de los "ensamblajes transnacionales" y, de acuerdo con Liliana Suárez (2006), de su interacción con los 
"campos" —el de la fe, el económico, el de la política-, sean éstos locales, nacionales o transnacionales. Una misma persona puede contender en campos distintos y a través de instancias diversas. Desde mi punto de vista, en el momento actual, mientras las instancias reticulares del ensamblaje transnacional libran batallas políticas como "sociedad civil migrante" en contra de la política de "securitización" - como la llama en este volumen Castro Neira-, las comunidades transnacionales rizomáticas absorben los costos económicos y sociales de las políticas demográficas y laborales que aquejan a quienes pertenecen a dichas comunidades.

\section{NUEVAS FORMAS DE GUBERNAMENTALIDAD O EL "HACER HACER" TRANSNACIONAL}

Si seguimos los planteamientos de Nancy Fraser, a la nueva configuración económica — posfordista, del capitalismo tardío o etapa de acumulación flexible, según el marco analítico que se use- corresponde una nueva forma de gobernanza y de gubernamentalidad. En la etapa anterior los sistemas de poder tenían claramente una escala nacional, hoy en día los procesos de gobernanza y las formas de gubernamentalidad toman una forma transnacional. El gobierno mexicano, por ejemplo, reconoce a su diáspora como parte constitutiva de la nación, construye fórmulas de atención a distancia y de participación en las elecciones, como el "voto de los mexicanos residentes en el extranjero". Hay una política y un aparato económico que producen a la mexicanidad en el contexto transnacional o que transnacionalizan al aparato de poder mexicano, sustentado en el nuevo régimen sentimental de la política y el mercado de la nostalgia. Es decir, a la transnacionalización de las comunidades siguió la transnacionalización del gobierno. Y para poder construir los dispositivos de control es necesario producir primero a los nuevos sujetos del poder: connacionales y trabajadores transnacionalizados. El Estado neoliberal se ha retirado de la vida de la población en muchos sentidos, nos dice Fraser, pero se han construido nuevos dispositivos de poder sobre ellos. Para algunos analistas, como Gupta y Sharma, la regulación social transnacional incluye ahora nuevos actores privados, como empresas y organizaciones no gubernamentales, que construyen la gubernamentalidad transnacional (Sharma y Gupta, 2006). Fraser añade otra dimensión, sugiere que el aparente retiro del Estado es en realidad una nueva fórmula en que los sujetos asumen mayores papeles y facultades de autogobierno. La autoayuda, la responsabilidad individual del bienestar, la salud, la búsqueda de empleo, la reunificación familiar, podríamos decir, hacen del posfordismo un régimen del "cuidado de sî" (Fraser, 2003). Rose y Miller agregan una tercera fórmula que resulta fundamental para comprender la situación de los transmigrantes entre México y Estados Unidos. Plantean que el nuevo modelo supone un nuevo sujeto de gobierno, no es más "lo social”, es el sujeto producido por el Estado para poder constituirse en un ente que impulsa el "bienestar social". La nueva forma de gubernamentalidad supuso la activación y la responsabilidad de las comunidades. Se trata de gobernar sobre comunidades que se autorregulen con la ayuda de una amplia red de tecnologías, sociales y materiales, que le permiten gravitar sobre los sistemas de valores, creencias y compromisos comunitarios. Estas comunidades se proveen a sí mismas de autoayuda y bienestar. Son comunidades morales - como las religiosas-, comunidades de compromiso -que se enfocan en problemas específicos, como la salud - o diaspóricas - como "las comunidades transnacionales indígenas” referidas por Kearney(Miller y Rose, 2008; Kearney, 1986). Si por un lado estamos ante mecanismos transnacionales de vinculación con el ensamblaje de espacios sociales transnacionales, como las comunidades, también es cierto que los sujetos son producidos como 
deportables. William Walters (2010) argumenta que desde el siglo xix la deportación se ha gubernamentalizado y que en el siglo XXI se extiende no sólo a sujetos cuya postura política es producida como políticamente indeseable, sino a distintas categorías de sujetos construidos como socialmente indeseables — "extranjeros", "criminales", "mano de obra excedente", etc.- Esta forma de gubernamentalidad se ha transformado en una modalidad de "exportación de los desempleados” (Strikwerda, 1997, citado en Walters, 2010). La deportabilidad es una "experiencia corporalizada", una forma inusual y vulnerable de "estar-en-el-mundo" que se vive como "anticipación", "experiencia" o se transforma en "memoria" tanto personal como colectiva ( $\mathrm{Ta}-$ lavera, Núñez-Mchiri y Heyman, 2010). Así, en las viñetas con que inicia este escrito los mixtecos de Tacache experimentaron la deportación, los familiares de Sandra Tafolla viven la deportación con anticipación y por ello regresan a los márgenes urbanos de los que partieron, mientras que los parientes del señor Mustafá se perciben como deportables, por lo que nunca viajan a España. Las tecnologías políticas que producen esta forma de gubernamentalidad son las leyes. Las tecnologías materiales que la concretan pueden ser las acciones en las fronteras y las "redadas", como las organizadas en los últimos años en distintas ciudades de Estados Unidos que han generado temor en las comunidades - aun entre los radicados en México, como en el caso de los habitantes de Tacache-. Los sujetos las incorporan con tecnologías del yo que responden a la percepción de que son sujetos "deportables" y con las que actúan en consecuencia - mecanismos cotidianos para evitar ser detenidos, cartografías del miedo de las ciudades donde viven y planes de retorno para escapar de ellas.

Con fineza etnográfica y agudeza analítica, las contribuciones de la sección "Saberes y razones" de este número de Desacatos nos permiten comprender cómo opera el régimen de deportación entre México y Estados Unidos en distintos niveles. Guillermo Alonso Meneses nos explica en primer lugar la historia de las leyes como tecnologías políticas que encuentran un correlato en las prácticas de acoso ciudadano y los discursos del racismo. La frontera como tecnología material se constituye en una frontera-gulag reforzada y acompañada de otras tecnologías, como las deportaciones en ciudades alejadas de la frontera. Estas tecnologías políticas y materiales tienen la posibilidad de detener y deportar, pero a la experiencia de la deportación - que el propio Alonso Meneses se pregunta si ha aumentado realmente - se suman la "anticipación" y la "memoria”, que se traducen en dinámicas poblacionales masivas sustentadas en gran parte en la renuncia de algunos a la movilidad y de otros a la movilidad en búsqueda de seguridad, basadas, suponemos — pero hace falta mayor investigación al respecto-, en tecnologías del yo que producen estrategias personales de respuesta en los habitantes de los ensamblajes transnacionales. Yerko Castro Neira nos ayuda a explicar que se trata no sólo de un sistema de tecnologías que opera en un sentido de imposición, sino de un "régimen" en el sentido de que es un aparato de gobierno que al mismo tiempo produce al gobierno. Es un sistema basado en la supuesta "seguridad nacional" responsable de la creación de zonas de excepción, desde las que se reproduce la inseguridad que justifica al "régimen”. Es una política del "dejar morir". Paralelo y cosustancial a este "régimen de deportación" aparece un aparato de gubernamentalidad que hace del ensamblaje un mecanismo de autocuidado. Luis Escala-Rabadán nos advierte que el reto que enfrentan las asociaciones y las comunidades transnacionales es ser cooptadas por los aparatos de gobierno, pero parece que el reto en realidad es que este gran ensamblaje transnacional asuma las responsabilidades de otras instancias, como el Estado y las empresas que les emplean. Desde este punto de vista se "deja vivir". Vistos en conjunto, el régimen de deportación y la nueva gubernamentalidad 


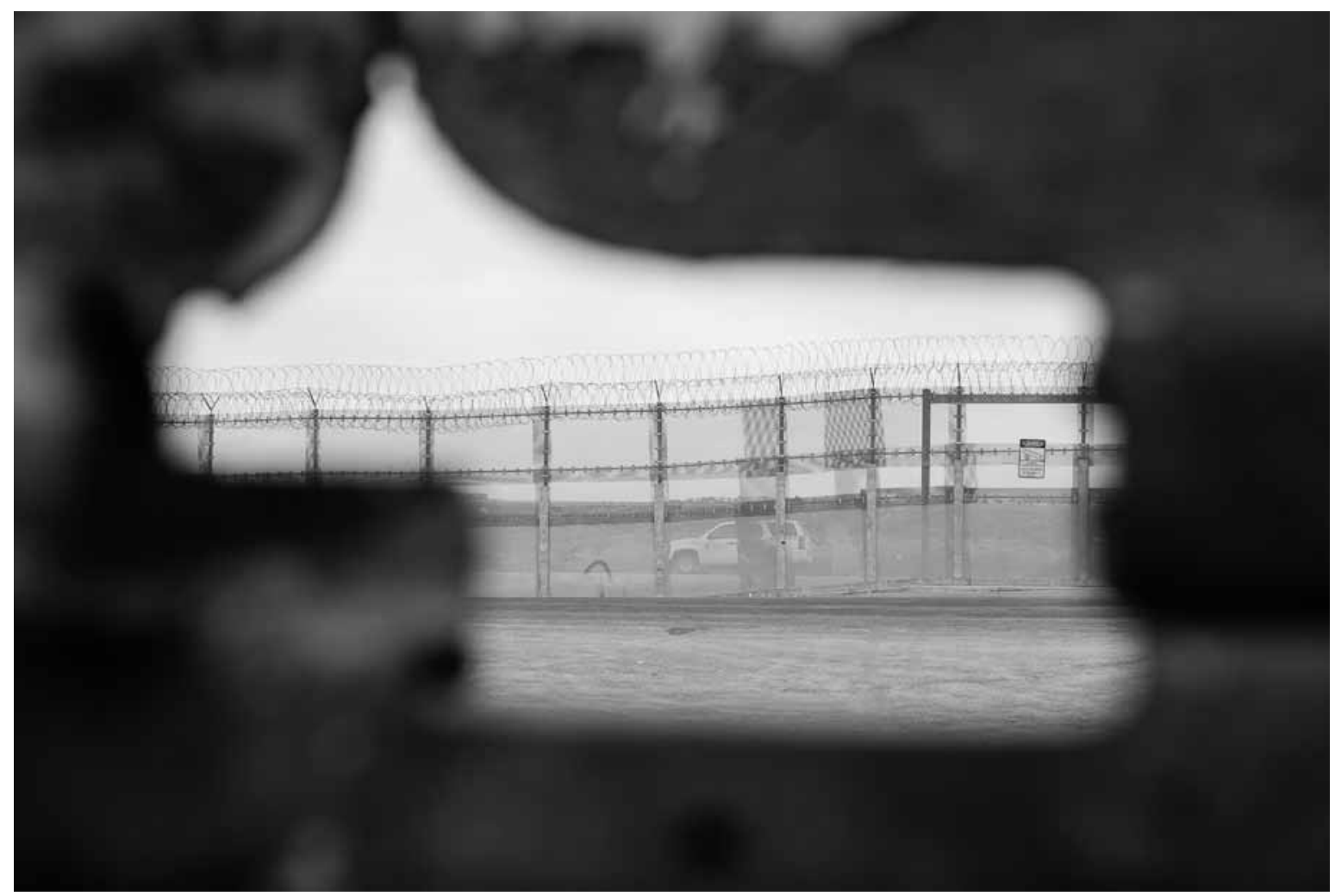

Prometeo Lucero Border Patrol, Tijuana.

transnacional son las dos caras de un mismo sistema de poder que podría resumirse en el principio de “dejar morir y dejar vivir".

\section{LOS NUEVOS ENSAMBLAJES DE LA VIOLENCIA Y EL PODER EN LOS ESPACIOS DE EXCEPCIÓN}

Necesitamos además construir una teoría de la violencia que explique las circunstancias cada vez más problemáticas que forman el contexto de la migración entre México y Estados Unidos. Un punto de inicio puede ser la discusión entre poder y violencia. Una perspectiva teórica propuesta por Arendt (2006) es que tenemos que pensar en el poder y la violencia como dos gestiones separadas. La violencia, por ejemplo, en Estados totalitarios, no permite la expresión del poder que reside en los sujetos. Es decir, la normalización de la violencia puede ser la condición para la no expresión del poder. El poder, que se expresa en acciones específicas de los sujetos, puede oponerse por ello a la violencia y pensarse a su vez en ausencia de la violencia. Otra manera de decirlo es que la violencia no es poder. En el Estado democrático el poder aparece atado a las formas de gubernamentalidad que "producen" poder desde los sujetos en sociedad para un Estado que lo administra - hacer vivir- La violencia se percibe como anómica, como "fuera de la sociedad" en espacios marginales considerados "antisociales". En el Estado totalitario la violencia se ejerce sobre "la sociedad" que aparece desempoderada - hacer morir- Si bien para Foucault el poder también emana de los sujetos, no es esporádico, sino que aparece 
continuamente, los aparatos de gubernamentalidad permiten su control, pero conviven con dispositivos por medio de los cuales se ejerce la violencia. En las condiciones actuales, los sistemas de gubernamentalidad operan sobre un sector que se encuentra crecientemente fuera de "lo social", estos nuevos "márgenes" son espacios de construcción de individuos y comunidades con agencia, "empoderados" como hemos visto, pero los ensamblajes sociales en estos espacios producen un poder fragmentado y son también espacio de violencia ejercida al mismo tiempo por el Estado y los grupos que podemos llamar de "violencias fácticas" — dejar vivir y dejar morir-. El caso descrito por Sandra Guillot de una niña que se "empodera" en contra de la violencia fáctica de su entorno más cercano describe con claridad esta complejidad. Ros, pese a su corta edad, actúa con agencia y toma sobre sí la tarea de producir el lazo social que la unirá con la madre y que las sacará de la violencia cotidiana de su barrio de origen, sólo para llegar a otro tipo de violencia estructural de carácter económico, donde continúa “cuidando de sí" y como un sujeto autodirigido. Los márgenes, nos han alertado Das y Poole (2004), son espacios de una gran productividad. Los márgenes pueden entenderse mejor como espacios de confluencia de dualidad, son lugares de producción, pero donde no operan los derechos, son lugares de control donde se deja a los sujetos a su suerte, son lugares donde aparecen los aparatos de gobierno, pero actúan en colusión con "violencias fácticas". Los márgenes como espacios de "excepción" son espacios característicos de la nueva condición transnacional: como zonas de producción para la exportación (Ong, 2006), zonas urbanas de autoconstrucción (Zirión, 2013), regiones de altísimo control del Estado como las fronteras donde los sujetos son abandonados a su suerte (Castro, en este volumen). Éstos, cada vez más, son los "terceros espacios" de la transnacionalidad y de la migración. En el caso de los migrantes podemos pensar en tres tipos de violencia específica que pasa por sus cuerpos. Una de ellas es lo que hemos llamado "violencia por aceleración" (Besserer y Gil, 2008), que se expresa en sus tres formas en el caso descrito por Sandra Guillot: el aceleramiento de los tiempos de vida que hace que sujetos que no han alcanzado la autonomía, como los niños, asuman responsabilidades que corresponden a la sociedad. El aceleramiento de la vida que reduce los tiempos de no trabajo y se expanden en el curso del día — con actividades que frecuentemente no están remuneradas - y en el curso de vida — como en el caso de los niños que se incorporan tempranamente a las responsabilidades de la sociedad-. La segunda forma, que puede inferirse de la anterior, es la paradójica fórmula que Sandra Guillot ha llamado "violencia por empoderamiento", que consiste en la transformación del sujeto en un actor en el proceso de vivir la expoliación y las carencias, con frecuencia sujetándose a sí mismo a tecnologías del yo que lejos de ser "cuidados de sí mismo" son violencia sobre sí mismo (Guillot, 2012).

Yerko Castro Neira propone enfocarnos en los "espacios de excepción" como una creación de la ley, en la medida en que la ley es una "productora de excepcionalidades". Su propuesta es que desde 2001 vivimos en una "era del terror" que ha ilegalizado y criminalizado a numerosos grupos en la sociedad, entre los que se encuentran los migrantes: arquetipo de la construcción de la exclusión, son hoy, en la era de la migración, una realidad que se produce todo el tiempo y que se vuelve invisible por su cotidianidad.

En ese sentido, los migrantes se han transformado en fantasmas. Como toda política, dice Castro Neira siguiendo las ideas de Benjamin, la política de la securitización se basa en la violencia, y la violencia se explica por la política, ahí la explicación de la violencia que hoy forma el entorno de la vida de los migrantes. El autor dice al inicio de su artículo que dejará de lado las "historias de éxito [...] que existen y aparecen de vez en cuando". Desde 
mi punto de vista, con este argumento deja fuera una discusión sobre el poder como la que tuvimos líneas más arriba. La nueva gubernamentalidad es una forma de movilización del poder que opera en estos espacios de excepción en contra de la población transmigrante, lo que se suma y profundiza la propuesta pesimista. Pero si la violencia y el poder están sujetos a las leyes de la dialéctica, entonces estos espacios de excepcionalidad, siguiendo las ideas de Patricia Fortuny y Marie Friedmann Marquardt y Luis Escala-Rabadán, son espacios de conflicto desde donde se producen también los movimientos contrahegemónicos y transformadores de la realidad. En el paisaje posapocalíptico en que se han constituido los territorios estadounidense y mexicano, los fantasmas de los transmigrantes suelen reaparecer — a la manera de la teoría fantasmagórica de Marx-como el "viejo topo" en la forma de actores dispuestos a cambiar la realidad.

\section{Conclusiones}

La migración entre México y Estados Unidos es un proceso que sucede en un nuevo momento, que se entiende mejor si lo analizamos en el marco de un nuevo modo de acumulación, de dinámicas demográficas que son el campo de acción de políticas públicas, donde la forma dominante de "lo social" está siendo sustituida por un "ensamblaje transnacional" sujeto a un nuevo sistema de formas de gubernamentalidad transnacional basadas en una nueva combinación de poder y violencia.

Los excelentes trabajos incluidos en la sección "Saberes y razones" de este número de Desacatos nos permiten sostener que desde finales del siglo xx comenzó un proceso de reforzamiento de la frontera y que con la crisis económica de 2008 aumentaron las deportaciones de los Estados Unidos hacia México. Esta crisis aparece en un contexto donde la producción y la política han creado "espacios de excepción” económica y jurídica, habitados por migrantes que configuran un gran ensamblaje de comunidades y asociaciones transnacionales. Las políticas de deportabilidad y la responsabilización de los migrantes sobre su propio bienestar se han articulado en un gran sistema de gubernamentalidad que permite la transferencia de los costos de la crisis a los migrantes y sus organizaciones e instituciones comunitarias. Esta forma de desposesión es de grandes dimensiones y se suma a un proceso demográfico que inició hace décadas, en el que las comunidades transnacionales se hacen cargo, además, de un sector creciente de su población en proceso de envejecimiento. Hemos pasado de una etapa en que los migrantes eran vistos como "herramientas para el desarrollo" a otra en la que son percibidos como "solucionadores de la crisis", a la vez que como agentes activos responsabilizados de su propio bienestar.

Los artículos que conforman la sección "Saberes y razones" de este número de Desacatos nos permiten hacer un análisis complejo de una realidad que se nos presenta como subordinante, violenta y devastadora. Sin embargo, los estudios aquí incluidos nos dejan observar en la experiencia cotidiana de los transmigrantes los indicios de su participación en la transformación de una realidad que ahora aparece ante nuestros ojos como una escena dantesca. D 


\section{Bibliografía}

Actor-Network Theory: Bruno Latour, Actant, Michel Callon, John Law, Aramis, or the Love of Technology, Annemarie Mol, Helen Verran, 2010, General Books LLc, Memphis.

Aguilar, Luz Adriana, 2012, Una transición sufrida: hacia la reconfiguración de la comunidad transnacional de Ixpantepec Nieves, Juan Pablos, Universidad Autónoma Metropolitana-Iztapalapa (Estudios Transnacionales), México.

Alba, Ximena, 2014, "El barrio transnacional”, en Federico Besserer y Daniela Oliver (eds.), Ensamblando la ciudad transnacional. Etnografía especular de los espacios transnacionales urbanos, Juan Pablos, Universidad Autónoma Metropolitana-Iztapalapa (Estudios Transnacionales), México, pp. 195-265.

Arendt, Hannah, 2006, Los orígenes del totalitarismo, Alianza, Madrid.

Besserer, Federico, 1986, "Nna Chka Ndavi. Internacionalización de la fuerza de trabajo y conciencia de clase en la comunidad mixteca migrante de San Juan Mixtepec", tesis de licenciatura en antropología, Universidad Autónoma Metropolitana-Iztapalapa, México.

__ y Michael Kearney (eds.), 2006, San Juan Mixtepec: una comunidad transnacional ante el poder clasificador y filtrador de las fronteras, Juan Pablos, Universidad Autónoma Metropolitana-Iztapalapa (Estudios Transnacionales), México.

__ y Rocío Gil, 2008, “¿Desde Babel o hacia Babel? Risomas y retículas en el mundo transmigrante”, en Paolo Corvo y Raúl Enríquez Valencia (eds.), Babele e dintorni. Fra catastrofismi e nuovi percorsi di senso, Polistampa, Florencia, pp. 79-87.

Castro Neira, Yerko, 2009, En la orilla de la justicia: migración y justicia en los márgenes del Estado, Juan Pablos, Universidad Autónoma Metropolitana-Iztapalapa (Estudios Transnacionales), México.

Consejo Nacional de Población (Conapo), 2002, La situación demográfica de México, 2002, Consejo Nacional de Población, México.

Das, Veena y Deborah Poole, 2004, Anthropology in the Margins of the State, School of American Research Press, Santa Fe.

Fraser, Nancy, 2003, “¿De la disciplina hacia la flexibilización? Releyendo a Foucault bajo la sombra de la globalización”, en Revista Mexicana de Ciencias Políticas y Sociales, vol. 46, núm. 187.

Fundación Bbva Bancomer, 2014, Anuario de migración y remesas. México 2014, Fundación BBva Bancomer, Secretaría de Gobernación, Consejo Nacional de Población, México.

Genova, Nicholas de y Nathalie Peutz (eds.), 2010, The Deportation Regime: Sovereignty, Space and the Freedom of Movement, Duke University Press, Durham.

Gil, Rocío, 2006, Fronteras de pertenencia. Hacia la construcción del bienestar y el desarrollo comunitario transnacional de Santa María Tindú, Oaxaca, Juan Pablos, Universidad Autónoma Metropolitana-Iztapalapa, Fundación Rockefeller (Estudios Transnacionales), México.

Guillot, Sandra, 2012, “Poder y violencia en la 'zona gris'. Un análisis de la situación de los niños salvadoreños migrantes en los espacios sociales transnacionales desde la antropología de las emociones", tesis de licenciatura en antropología, Universidad Autónoma Metropolitana-Iztapalapa, México.

Harvey, David, 2008, "The Right to the City”, en New Left Review, núm. 53.

Herrera, Fernando, Óscar Calderón y Leticia Hernández, 2006, "Redes que comunican y redes que enclaustran: evidencia de tres circuitos migratorios contrastantes", ponencia, Segundo Coloquio Internacional sobre Migración y Desarrollo: Migración, Transnacionalismo y Transformación Social, 26-28 de octubre, Morelos.

Herrera, Gioconda, 2005, "Mujeres ecuatorianas en las cadenas globales de cuidado”, en Gioconda Herrera, María Cristina Carrillo y Alicia Torres (eds.), La migración ecuatoriana. Transnacionalismo, redes e identidades, Facultad Latinoamericana de Ciencias SocialesEcuador, Plan Migración, Comunicación y Desarrollo, Quito.

Hirai, Shinji, 2009, Economía política de la nostalgia. Un estudio sobre la transformación del paisaje urbano en la migración transnacional entre México y Estados Unidos, Universidad Autónoma Metropolitana-Iztapalapa, México.

Instituto Nacional de Estadística y Geografía (INEGI), 2011a, Principales resultados del Censo de Población y Vivienda 2010, Instituto Nacional de Estadística y Geografía, México.

, 2011b, Censo de Población y Vivienda 2010, Instituto Nacional de Estadística y Geografía, México.

Kearney, Michael, 1986, "From the Invisible Hand to Visible Feet: Anthropological Studies of Migration and Development”, en Annual Review of Anthropology, núm. 15, pp. 331-361.

—_ y Carole Nagengast, 1989, "Anthropological Perspectives on Transnational Communities in Rural California”, Working Group on Farm, Labor and Rural Poverty-California Institute for Rural Studies (Working Paper, núm. 3), California. 
Lara, Tatiana, 2014, “La colonia transnacional”, en Federico Besserer y Daniela Oliver (eds.), Ensamblando la ciudad transnacional. Etnografía especular de los espacios transnacionales urbanos, Juan Pablos, Universidad Autónoma Metropolitana-Iztapalapa (Estudios Transnacionales), México, pp. 63-127.

Levitt, Peggy y Nina Glick Schiller, 2004, "Conceptualizing Simultaneity: A Transnational Social Fields Perspective on Society”, en International Migration Review, vol. 38, núm. 3, pp. 595-629.

Marx, Karl, 2003, El dieciocho Brumario de Luis Bonaparte, Alianza, Madrid.

Miller, Peter y Nikolas Rose, 2008, Governing the Present: Administering Economic, Social and Personal Life, Polity, Cambridge.

Moctezuma, Miguel, 2011, La transnacionalidad de los sujetos. Dimensiones, metodologías y prácticas convergentes de los migrantes mexicanos en Estados Unidos, Universidad Autónoma de Zacatecas, Miguel Ángel Porrúa, México.

Oliver, Daniela y Cristian Torres, 2012, Excluidos y ciudadanos. Las dimensiones del poder en una comunidad transnacional mixteca, Juan Pablos, Universidad Autónoma Metropolitana-Iztapalapa (Estudios Transnacionales), México.

Ong, Aihwa, 2006, Neoliberalism as Exception: Mutations in Citizenship and Sovereignty, Duke University Press, Durham.

Ordaz Díaz, Juan Luis y Juan José Li Ng, 2012, "Flash Migración México: Ios migrantes rejuvenecen a los Estados Unidos”, Fundación BBva Bancomer-Servicio de Estudios Económicos-México bBva Research, 25 de junio, México.

Pizarro, Karina, 2010, El pasaporte, la maleta y la barbacoa: la experiencia urbana a través de los saberes y sabores transnacionales PachucaChicago, Instituto de Ciencias Sociales y Humanidades-Universidad Autónoma del Estado de Hidalgo, Pachuca.

Pries, Ludger, 2002, New Transnational Social Spaces: International Migration and Transnational Companies in the Early Twenty-first Century, Taylor and Francis, Londres.

Reyes, Daniela, 2014, "La comunidad indígena urbana transnacional”, en Federico Besserer y Daniela Oliver (eds.), Ensamblando la ciudad transnacional. Etnografía especular de los espacios transnacionales urbanos, Juan Pablos, Universidad Autónoma MetropolitanaIztapalapa (Estudios Transnacionales), México, pp. 129-193.

Rivera, Liliana, 2012, Vinculos y prácticas de interconexión en un circuito entre México y Nueva York, Consejo Latinoamericano de Ciencias Sociales (Colección Becas de Investigación), Buenos Aires.

Rouse, Roger, 1991, "Mexican Migration and the Social Space of Postmodernism”, en Diaspora. A Journal of Transnational Studies, vol. 1, núm. 1. Serrano Herrera, Carlos (coord.), 2014, Anuario de migración y remesas 2014, Consejo Nacional de Población, Fundación BBva-Bancomer, México. Sharma, Aradhana y Akhil Gupta, 2006, The Anthropology of the State. A Reader, Blackwell, Oxford.

Smith, Robert Courtney, 2008, "Contradictions of Diasporic Institutionalization in Mexican Politics: The 2006 Migrant Vote and other Forms of Inclusion and Control", en Ethnic and Racial Studies, vol. 31, núm. 4, pp. 708-741.

Solís, Lilia, en prensa, "Manhattan para los mixtecos. Una comunidad transnacional mixteca en los márgenes de la ciudad global”, en Federico Besserer y Raúl Nieto (eds.), La ciudad transnacional comparada.

Suárez, Liliana, 2006, “Un nuevo actor migratorio: jóvenes, rutas y ritos juveniles transnacionales”, en Francisco Checa, Ángeles Arjona y Juan Carlos Checa Olmos (eds.), Menores tras la frontera. Otra inmigración que aguarda, Icaria, Barcelona, pp. 17-50.

Tafolla, Sandra, 2014, "La calle transnacional”, en Federico Besserer y Daniela Oliver (eds.), Ensamblando la ciudad transnacional. Etnografía especular de los espacios transnacionales urbanos, Juan Pablos, Universidad Autónoma Metropolitana-Iztapalapa (Estudios Transnacionales), México, pp. 43-62.

Talavera, Víctor, Guillermina Gina Núñez-Mchiri y Josiah Heyman, 2010, “Deportation in the U. S.-Mexico Borderlands: Anticipation, Experience, and Memory", en Nicholas de Genova y Nathalie Peutz (eds.), The Deportation Regime: Sovereignty, Space, and the Freedom of Movement, Duke University Press, Durham.

Walters, William, 2010, "Deportation, Expulsion, and the International Police of Aliens", en Nicholas de Genova y Nathalie Peutz (eds.), The Deportation Regime: Sovereignty, Space and the Freedom of Movement, Duke University Press, Durham.

Wence, Nancy, 2012, El pequeño gobierno: una comunidad transnacional mixteca en la lucha por conservar su gobernabilidad, Comisión Nacional para el Desarrollo de los Pueblos Indígenas, Juan Pablos, Universidad Autónoma Metropolitana (Estudios Transnacionales), México.

__ en prensa, "Economía política de una ciudad diaspórica entre el Atlántico y las Américas", en Federico Besserer y Raúl Nieto (eds.), La ciudad transnacional comparada.

Wolf, Eric, 1955, “Types of Latin American Peasantry: A Preliminary Discussion”, en American Anthropologist, vol. 57, núm. 3, pp. $452-471$. y Sidney Mintz, 1957, "Haciendas and Plantations in Middle America and the Antilles", en Social and Economic Studies, vol. 6, núm. 3, pp. 380-412.

Zirión, Antonio, 2013, La construcción del habitar. Transformación del espacio y la cultura albañil en la ciudad de México a principios del siglo xxI, Juan Pablos, Universidad Autónoma Metropolitana-Iztapalapa, México. 